\title{
Radar Emitter Classification Based on a Multiperspective Collaborative Clustering Method and Radar Characteristic Spectrum
}

\author{
Jundi Wang $\mathbb{D}^{1}{ }^{1}$ Xing Wang, ${ }^{1}$ Yipeng Zhou, ${ }^{2}$ and You Chen ${ }^{1}$ \\ ${ }^{1}$ Aviation Engineering School, Air Force Engineering University, 1 Baling Road, Xi'an 710038, China \\ ${ }^{2}$ Aviation University Air Force, Changchun, Jilin, China \\ Correspondence should be addressed to Jundi Wang; qxwangjundi@sina.com
}

Received 8 October 2021; Accepted 27 January 2022; Published 28 February 2022

Academic Editor: Xingling Shao

Copyright (c) 2022 Jundi Wang et al. This is an open access article distributed under the Creative Commons Attribution License, which permits unrestricted use, distribution, and reproduction in any medium, provided the original work is properly cited.

\begin{abstract}
Modern cognitive electronic reconnaissance methods for radar systems must contend with the complex electromagnetic environments arising from the deployment of multiple signal sources and radar countermeasures, which greatly limit access to the degree of prior information required to enable effective target recognition. The present work addresses this issue by proposing a multiperspective collaborative clustering method for sorting radiation sources based on the multiperspective information of radar signals. In contrast to conventional collaborative training approaches, which are suitable only for semisupervised learning, the proposed multiperspective collaborative clustering method performs unsupervised clustering, cluster label transfer, and dimensionality reduction by linear discriminant analysis iteratively based on the differences between the clustering results obtained from two signal perspectives radiation signal sorting can be conducted in a noncooperative context. The results of comparative experiments demonstrate that the proposed multiperspective sorting method can make full use of the difference information between basic signal characteristics and intrapulse features and thereby improve the accuracy of clustering-based radiation source sorting. Accordingly, the sorting ability of the proposed method is superior to those of other state-of-the-art clustering methods and that of the single-perspective clustering-based sorting method.
\end{abstract}

\section{Introduction}

The rapid development of information technology in recent years has made electronic warfare a key factor in victory at war [1]. In this regard, electronic countermeasures, such as radar countermeasures, have become a principal component of electronic warfare that has been proven to be particularly important in air-to-air combat, where these countermeasures directly affect the completion of combat missions and protect the lives and safety of aircraft pilots. The accurate sorting and recognition of radar signals is a prerequisite for managing the complexity of the electromagnetic environment in the battlefield arising from the use of radar countermeasures. Moreover, these activities provide a reference for subsequent threat assessment and the rational use of jamming resources.
The increasingly complex electromagnetic environment of the battlefield and the continuous development of radar technology have promoted a transition from singleparameter radar signal sorting methods to multiparameter sorting. Moreover, the development of multiparameter sorting methods has transitioned from template matching methods to comprehensive sorting based on clustering in recent years. In 1989, Mardia [2] proposed radar signal sorting based on a sequence search and proposed the cumulative difference (CDIF) histogram algorithm. In 1993, Nelson [3] first proposed the pulse repetition interval (PRI) transform algorithm, which improved the autocorrelation function by increasing the rotation factor and improved the PRI extraction method based on the time of arrival (TOA) sequence of complex autocorrelation integral peaks. In 1992, Chandra and Bajpai [4] proposed a two-stage clustering and sorting 
algorithm based on pulse description words (PDWs). Expectation-based maximization clustering algorithm [5], K-modes [6], and Density-Based Spatial Clutering of Application with Noise (DBSCAN) [7] are widely used in radar signal sorting.

The basic characteristics of radar signals include the parameters carrier frequency, pulse width, pulse amplitude, pulse angle of arrival, and pulse TOA. These five basic parameters can be directly measured by radar signal receivers. However, the wide application of low intercept technology and parameter agility technology in modern phased array radar systems has increased the pulse density significantly, and the problems associated with pulse loss and pulse overlap have become increasingly serious [8-11]. As a result, conventional radar signal characterization methods based on pulse description parameters suffer from coarse information granularity and insufficient discrimination. These issues make the effective separation of phased array radar signals difficult to achieve using conventional signal sorting methods based on pulse descriptors. This has prompted an urgent need for the development of new signal features. For example, Klein et al. [12, 13] used the cutting and segmentation method to calculate the statistical feature vector of the instantaneous sequence. They selected statistical features, including variance, skewness, and kurtosis, and named this statistical feature vector RF-DNA (replicative form deoxyribo cucleic acid). Subsequently, Dudczyk and Kawalec [14-16] proposed the use of fractal dimensions to extract intrapulse features based on multifractal theory.

The recent development of artificial intelligence technology has promoted the application of machine learning algorithms to the analysis of radar signals. Haykin $[17,18]$ first proposed the concept of cognitive radar, which was progressively transformed from the artificial cognition process of machines to an autonomous cognition process requiring no human intervention. In some cases, the process for recognizing radar emitter signals has been conducted using unsupervised learning algorithms such as the k-means clustering [19], the C-means clustering [20], the ambiguity function [21], and the density clustering algorithm [22]. However, while these methods are relatively simple and computationally efficient, the final recognition result is not ideal because these algorithms cannot adequately label the signal categories. Other approaches have obtained better signal recognition results by applying machine learning algorithms such as extreme learning machines [23], support vector machines [24], neural networks [25-28], and Bayesian programming [29]. However, the learning process of these algorithms requires a large volume of prior information, which is greatly restricted in the complex electromagnetic environment prevalent on modern battlefields [30]. Moreover, the noncooperative identification process inevitably introduces inaccuracies in the signal recognition results.

This issue has been addressed by the development of the collaborative training method. Here, Blum and Mitchell [31] proposed a standard collaborative training method for conducting machine learning from two sufficiently independent perspectives. This method has been subsequently developed by relaxing some of the assumptions required in the stan- dard method, which has enabled the collaborative training method to be conducted under conditions where the perspectives are not independent of conditions [32, 33]. This has led to the development of multiperspective clustering, which integrates the complementarity, difference, and consistency information of multiple signal features derived for a clustering target. The use of multiperspective clustering methods has been demonstrated to improve the signal sorting ability of modern cognitive electronic reconnaissance systems for radar signals with characteristics obtained from two perspectives, including basic signal characteristics and intrapulse features. However, the presently available collaborative training methods are only applicable for learning multiperspective data with partial labels. Therefore, these methods are only suitable for semisupervised learning, which greatly restricts the level of automation required for conducting cognitive electronic reconnaissance in complex electromagnetic environments. Moreover, multiperspective data suffers from high dimensionality and is therefore subject to common problems, such as the appearance of equidistant data, making the clustering of data problematic [34, 35]. Therefore, dimensionality reduction is necessary. Nonetheless, the nonlinear characteristics of the distributions in the intrapulse feature data of radar signals make the application of the standard principal component dimensionality reduction method problematic.

The present work addresses this issue by proposing a radar signal sorting and recognition method based on a multidimensional feature expression system and a multiperspective clustering method. Inspired by the multiperspective discriminative clustering algorithm, the proposed method also takes into account the characteristics of radar signals and the noncooperative nature of electronic reconnaissance. In addition, a collaborative training framework is adopted, which combines multiple perspectives, including the basic characteristics of radar signals and intrapulse feature data. The convergence of the clustering process is achieved by iteratively conducting unsupervised clustering and cluster label transfer. In addition, we expand the radar signal characteristic system and increase the dimension of radar signal characteristic expression by extracting the instantaneous and statistical characteristics of radar signals. Finally, we apply the kernel principal component analysis (KPCA) method to reduce the dimensionality of the characteristic spectrum of radar signals. Application of the multiperspective clustering method to actual radar signals in a realistically complex electromagnetic environment is demonstrated to improve the ability of cognitive electronic reconnaissance systems to sort radar signals.

\section{Composition of Radar Characteristic Spectrum}

2.1. Instantaneous Characteristics. Due to the differences in signal modulation methods, unintentional modulations of different radiation sources, and different propagation paths of signals to reconnaissance receivers, the instantaneous characteristics of signals from different radiation sources are significantly different. Therefore, the signals can be 
distinguished by extracting the instantaneous features of the signals to realize signal sorting.

For a detection signal $s(n), n=1,2, \cdots, N$, and the analytical form of the signal after applying the Hilbert transform is

$$
s(n)=I(n)+j Q(n)
$$

where $I(n)$ and $Q(n)$ are the respective instantaneous inphase and quadrature components of the signal. Therefore, the instantaneous amplitude $a(n)$, instantaneous phase $\phi(n$ ), and instantaneous frequency $f(n)$ of the signal are given as follows.

$$
\begin{aligned}
& a(n)=\sqrt{I(n)^{2}+Q(n)^{2}}, \\
& \phi(n)=\arctan \frac{Q(n)}{I(n)}, \\
& f(n)=\frac{1}{2 \pi} \frac{\phi(n)-\phi(n-1)}{\Delta n} .
\end{aligned}
$$

In practical applications, the impact of environmental noise and receiver equipment tolerances must be reduced. Therefore, $a(n), \varphi(n)$, and $f(n)$ must be given with respect to their mean values (i.e., centralized). The centralization processing applied for $a(n)$ and $f(n)$ is given as follows.

$$
\begin{aligned}
& a_{c}(n)=a(n)-\mu_{a}, \\
& f_{c}(n)=f(n)-\mu_{f} .
\end{aligned}
$$

Here, $\mu_{a}$ and $\mu_{f}$ are the mean values of the instantaneous amplitude series and instantaneous frequency series, respectively. For $\phi(n)$, the nonlinear phase distortion caused by the frequency estimation error in the receiver down-conversion process must be removed before conducting centralization. This nonlinear phase distortion can be expressed as

$$
\phi_{n l}(n)=\phi(n)-2 \pi \mu_{f}(n) \Delta t
$$

where $\Delta t$ is the sampling interval. Therefore, the centralized instantaneous phase $\varphi_{c}(n)$ can be obtained on the basis of formula (4) as follows:

$$
\phi_{c}(n)=\phi_{n l}(n)-\mu_{\phi n l}
$$

where $\mu_{\phi n l}$ is the mean value of $\phi_{n l}(n)$. After the centralization process, the instantaneous features are normalized as follows.

$$
\begin{aligned}
A(n) & =\lg \left(a_{c}(n)\right)-\max \left(\lg \left(a_{c}(n)\right)\right), \\
P(n) & =\phi_{c}(n) / \max \left|\phi_{c}(n)\right|, \\
F(n) & =\frac{1}{2 \pi} \frac{\phi(n)-\phi(n-1)}{\Delta n} .
\end{aligned}
$$

However, the instantaneous feature dimensions are relatively high after centralization and normalization, and this will produce feature redundancy if used directly for signal sorting. Therefore, we apply statistical features, including variance, skewness, and kurtosis, as proposed in a previous study [29]. To this end, we assume that $A(n), P(n)$, and $F(n)$ are divided into $R$ segments $x$ of length $N_{x}$. For the $i$ -th sequence segment $x(k)$, we calculate its variance $\sigma^{2}$, skewness $\gamma$, and kurtosis $\kappa$ according to the mean value $a$ in a sequence segment as follows.

$$
\begin{aligned}
& \sigma_{x}^{2}=\frac{1}{N_{x}} \sum_{k=1}^{N_{x}}[x(k)-\bar{x}]^{2}, \\
& \gamma_{x}=\frac{1}{N_{x}} \sum_{k=1}^{N_{x}}[x(k)-\bar{x}]^{3}, \\
& \kappa_{x}=\frac{1}{\sigma_{x}^{4} N_{x}} \sum_{k=1}^{N_{x}}[x(k)-\bar{x}]^{4} .
\end{aligned}
$$

Therefore, the statistical characteristics of the $i$-th instantaneous feature are

$$
\mathrm{T}_{\mathrm{i}}=\left[\sigma_{i}^{2} \gamma_{i} \kappa_{i}\right]
$$

2.2. Bispectral Characteristics. The high-order spectrum of a signal is a $k-1$ dimensional Fourier transform of the $k$-th order cumulant of the signal $[13,36]$. The high-order spectrum contains the phase and amplitude information of the signal and has the characteristics of time-shift invariance, scale variability, and phase retention. A bispectrum represents the third-order spectrum in the high-order spectrum of a signal, which can be obtained by applying a twodimensional Fourier transform to the third-order cumulant of the signal. The bispectrum of a signal is particularly useful because it contains phase information that is not available in second-order statistics, such as the power spectrum. Moreover, the use of a bispectrum is more convenient than other high-order spectra because the bispectrum operation can better avoid the common problems associated with high dimensionality. [37, 38]

For a radiation source signal $x(t)$, where $t$ is time, its bispectrum is given as

$$
B_{x}\left(w_{1}, w_{2}\right)=G_{3, x}\left(w_{1}, w_{2}\right)=\sum_{\tau_{1}} \sum_{\tau_{2}} C_{3 x}\left(\tau_{1}, \tau_{2}\right) e^{-j\left(w_{1} \tau_{1}+w_{2} \tau_{2}\right)},
$$

where $C_{3 x}\left(\tau_{1}, \tau_{2}\right)$ is the third-order cumulant of $x(t), w_{1}$ and $w_{2}$ are the signal frequency, respectively, and $\tau_{1}$ and $\tau_{2}$ are time, respectively. Here, $C_{3 x}\left(\tau_{1}, \tau_{2}\right)$ is defined as follows:

$$
C_{3 x}\left(\tau_{1}, \tau_{2}\right)=E\left\{x^{*}(t) x\left(t+\tau_{1}\right) x\left(t+\tau_{2}\right)\right\}
$$

where $E\{\cdot\}$ is the mathematical expectation and $x^{*}(t)$ is the signal transpose.

Two nonparametric methods can be applied for signal bispectrum estimation, which include the indirect method and direct method. Here, we apply the indirect method, 
and the statistical features and entropy features of the integral bispectrum are calculated by cutting segmentation. The statistical characteristics of the integral bispectrum include variance, skewness, and kurtosis, while the entropy features of the integral bispectrum include permutation entropy and fuzzy entropy.

As discussed above, we assume that the integral bispectrum of the signal is divided into $R$ segments. We then calculate the variance, skewness, kurtosis, permutation entropy $\left(\operatorname{PerE} n_{i}\right)$, and fuzzy entropy $\left(F u E n_{i}\right)$ of the $i$-th sequence segment $x(i)$ of the integral bispectrum to obtain the following bispectral eigenvector:

$$
\begin{gathered}
\operatorname{PerEn}(m)=-\sum_{j=1}^{k} P_{j} \ln \left(P_{j}\right), \\
\mathbf{T}_{i}=\left[\sigma_{i}^{2} \gamma_{i} \kappa_{i} \operatorname{PerEn}_{i} F u E n_{i}\right]_{1 \times 5},
\end{gathered}
$$

where $P_{i}$ is the probability of each sequence and $N_{x}$ is the signal sequence length.

The feature vector composed of the feature vectors of all segments is

$$
\mathbf{T}=\left[\mathbf{T}_{1}: \mathbf{T}_{2}: \mathbf{T}_{3} \cdots \mathbf{T}_{R+1}\right]_{1 \times 5(R+1)} .
$$

Finally, the signal bispectrum statistical feature set is obtained according to the order of axial integral bispectrum (AIB), circular integral bispectrum (CIB), radial integral bispectrum (RIB), and contour integral bispectrum (SIB), as follows:

$$
\mathbf{T}=\left[\mathbf{T}^{A I B}: \mathbf{T}^{C I B} \vdots \mathbf{T}^{R I B} \vdots \mathbf{T}^{S I B}\right]_{1 \times 5(R+1) \times 4} .
$$

The proposed bispectral feature extraction process is presented in Figure 1.

According to Figure 1, the specific steps for extracting the bispectral feature vector of the signal are given as follows:

(1) Bispectrum estimation of the radar signal: apply the indirect method to estimate the bispectral plane of the signal

(2) Extraction of integral bispectrum: extract the integral bispectral sequence according to the integral path

(3) Cutting segmentation and feature extraction of bispectral sequence: divide the integral bispectrum of the signal into $R$ segments and calculate the statistical characteristics and entropy characteristics of each bispectrum sequence according to formulas (11)-(17)

(4) Signal bispectrum feature set: combine the statistical features and entropy features of the signals into a signal bispectral feature set according to the order AIB, CIB, RIB, and SIB

\section{Dimensionality Reduction of Intrapulse Features Based on KPCA}

For a single radar pulse signal, the instantaneous feature parameters are 60 dimensions, and the integral bispectral feature parameters are 80 dimensions. Therefore, the intrapulse feature parameters attain a relatively high data dimensionality of 140 dimensions. This can be addressed by noting that the different characteristics in the radar spectrum are not strictly independent and by taking account of the nonlinear distribution of the characteristics in a radar signal pulse. The present work conducts this process by applying the KPCA method.

The basic process of KPCA dimensionality reduction firstly maps the original data to a high-dimensional space through a nonlinear mapping function. Then, linear principal component analysis (PCA) is applied to the highdimensional space to achieve dimensionality reduction. The specific process is given as follows.

For a dataset $D=\left\{\boldsymbol{x}_{1}, \boldsymbol{x}_{2}, \cdots, \boldsymbol{x}_{n}\right\} \in R^{m \times n}$, where $m$ is the number of features and $n$ is the number of data samples, applying a nonlinear mapping transformation $\varphi$ maps the data from the original $R^{m \times n}$ feature space to the highdimensional feature space $F$ :

$$
\varphi: R^{m \times n} \longrightarrow F, x \longrightarrow X
$$

The transformed feature vector data in $F$ can be expressed as a linear combination of $\varphi\left(\boldsymbol{x}_{1}\right), \varphi\left(\boldsymbol{x}_{2}\right), \cdots, \varphi\left(\boldsymbol{x}_{n}\right)$ and $\sum_{i=1}^{n} \varphi\left(x_{i}\right)=0$ because the data samples in the highdimensional feature space are centralized. Therefore, the covariance matrix in the transformed high-dimensional feature space is given as follows.

$$
\boldsymbol{C}^{F}=\frac{1}{n} \sum_{i=1}^{n} \varphi\left(\boldsymbol{x}_{i}\right) \varphi\left(\boldsymbol{x}_{i}\right)^{T}=\frac{1}{n} \varphi(\boldsymbol{X}) \varphi(\boldsymbol{X})^{T}
$$

The fact that the feature vector in $F$ can be expressed as a linear combination of $\varphi\left(\boldsymbol{x}_{1}\right), \varphi\left(\boldsymbol{x}_{2}\right), \cdots, \varphi\left(\boldsymbol{x}_{n}\right)$ enables the application of the inner product rule $(\boldsymbol{X} \cdot \boldsymbol{Y})=\boldsymbol{X}^{T} \boldsymbol{Y}$ to obtain the following equation:

$$
\lambda\left(\varphi\left(\boldsymbol{x}_{k}\right) \cdot \boldsymbol{v}\right)=\left(\varphi\left(\boldsymbol{x}_{k}\right) \cdot \boldsymbol{C}^{F} \boldsymbol{v}\right)
$$

Among these terms, $\boldsymbol{v}$ is the eigenvector of $\boldsymbol{C}^{F}$ and is given as $\boldsymbol{v}=\sum_{i=1}^{n} \alpha_{i} \varphi\left(\boldsymbol{x}_{k}\right)$, and $\lambda$ is the eigenvalue of $\boldsymbol{C}^{F}$. Therefore, (16) can be rewritten as

$$
\lambda \sum_{i=1}^{n} \alpha_{i}\left(\varphi\left(\boldsymbol{x}_{k}\right) \cdot \varphi\left(\boldsymbol{x}_{i}\right)\right)=\frac{1}{n} \sum_{i=1}^{n} \alpha_{i}\left(\varphi\left(\boldsymbol{x}_{k}\right) \cdot \sum_{j=1}^{n} \varphi\left(\boldsymbol{x}_{j}\right)\right)\left(\varphi\left(\boldsymbol{x}_{j}\right) \cdot \varphi\left(\boldsymbol{x}_{i}\right)\right) .
$$

Defining an $n \times n$ matrix $\boldsymbol{K}_{i j}=\varphi\left(\boldsymbol{x}_{i}\right) \cdot \varphi\left(\boldsymbol{x}_{j}\right)$ enables (17) to be simplified as

$$
n \lambda \boldsymbol{K} \alpha=K^{2} \boldsymbol{\alpha},
$$




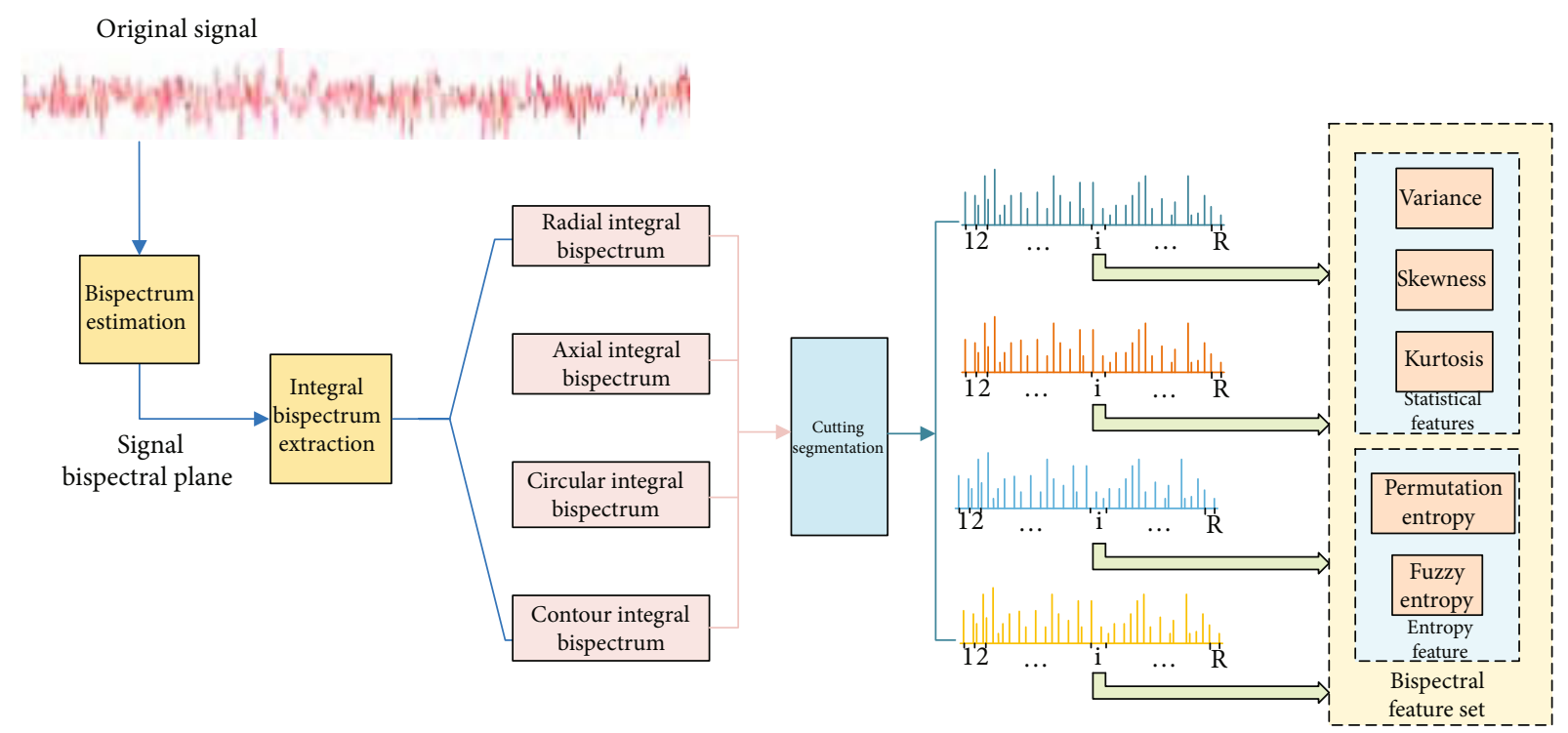

Figure 1: Bispectral feature vector extraction process.

and because $\boldsymbol{K}$ is symmetric, (18) can be simplified as

$$
n \lambda \alpha=K \alpha
$$

Therefore, $n \lambda$ and $\boldsymbol{\alpha}$ can be solved according to (19).

For the eigenvector $\boldsymbol{v}$, we note that $\boldsymbol{v} \cdot \boldsymbol{v}^{T}=1$. This, in addition to the given definition of $v$, yields the following.

$$
\begin{aligned}
\left(\boldsymbol{v} \cdot \boldsymbol{v}^{T}\right) & =\sum_{i, j=1}^{n} \alpha_{i}^{k} \alpha_{j}^{k}\left(\varphi\left(\boldsymbol{x}_{i}\right) \cdot \varphi\left(\boldsymbol{x}_{j}\right)\right) \\
& =\sum_{i, j=1}^{n} \alpha_{i}^{k} \alpha_{j}^{k} K\left(\boldsymbol{x}_{i}, \boldsymbol{x}_{j}\right)=\lambda_{k}\left(\alpha^{k} \alpha^{k}\right)=1 .
\end{aligned}
$$

The dimensionality of the data in $F$ is then reduced by calculating the projection of the sample on the feature vector $\boldsymbol{v}$ in $F$. This is obtained for sample $\boldsymbol{x}$ and its transformation $\varphi(\boldsymbol{x})$ in $F$ as follows:

$$
\left(\boldsymbol{v}^{k} \cdot \varphi(\boldsymbol{x})\right)=\sum_{i=1}^{n} \alpha_{i}^{k}\left(\varphi\left(\boldsymbol{x}_{i}\right) \cdot \varphi(\boldsymbol{x})\right) .
$$

Accordingly, the principal components of the sample can be obtained.

The kernel function employed in KPCA can be any symmetric function satisfying Mercer's theorem. A number of kernel functions are commonly employed, such as polynomial kernel functions, Gaussian kernel functions, and neural network kernel functions, which are, respectively, given in the following equations.

$$
\varphi(\boldsymbol{x}) \cdot \varphi(\boldsymbol{y})=\exp \left(-\frac{\|\boldsymbol{x}-\boldsymbol{y}\|}{2 \sigma^{2}}\right) .
$$

The present work adopts the Gaussian kernel function in (23) as the mapping function.

\section{Multiperspective Collaborative Clustering and Sorting Algorithm}

The basic process of the proposed multiperspective collaborative clustering and sorting algorithm is presented in Figure 2, and Algorithm 1 presents its detailed processing steps.

The proposed multiperspective collaborative clustering and sorting method mainly offers the following important advantages:

(1) The preprocessing stage focuses on the basic characteristics of the signal and the intrapulse feature data, and PCA and KPCA are, respectively, applied for data dimensionality reduction. It should be noted that the basic signal characteristics data employed in the simulation experiments in this article are of generally low dimensionality (i.e., 3 dimensions). Therefore, the dimensionality of this data remains unchanged during the actual processing, and only the dimensionality of the intrapulse feature data is reduced using the KPCA method. This processing enhances the discrimination between the characteristics of different radiation sources because, on the one hand, it can achieve data dimensionality reduction while maintaining the nonlinear characteristics of the data as much as possible. On the other hand, it can increase the impact of core features while reducing the impact of redundant features. In addition, data dimensionality reduction can also reduce the calculational burden in the subsequent clustering process

(2) The multiperspective collaborative clustering and sorting method learns data from different perspectives alternately and uses the difference between the 


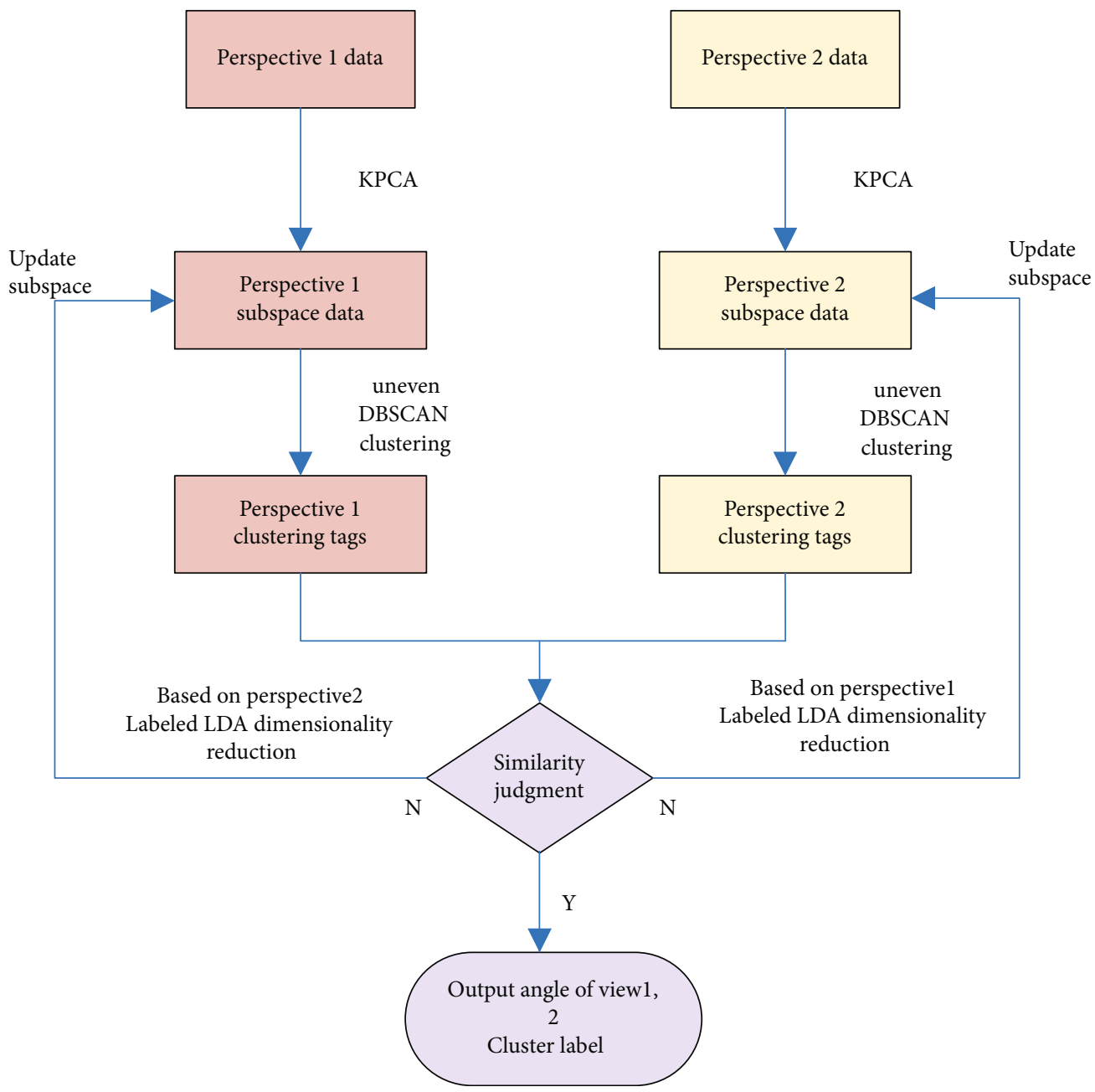

FiguRE 2: Basic process of the multiperspective collaborative clustering and sorting method.

Input: Perspective 1 basic signal characteristic data $\boldsymbol{T}^{(1)}$ and perspective 2 intrapulse feature data $\boldsymbol{T}^{(2)}$

Output: Cluster label $\boldsymbol{L}$ (data label $\boldsymbol{L}^{(1)}$ for perspective 1 and data label $\boldsymbol{L}^{(2)}$ for perspective 2)

Steps:

Step 1. Apply KPCA dimensionality reduction to the basic signal characteristic data of perspective 1 to obtain subspace $\boldsymbol{T}^{(1)}$, and apply KPCA dimensionality reduction to the intrapulse feature data of perspective 2 to obtain subspace $T^{(2)}$.

Step 2. Sort subspaces $\boldsymbol{T}^{(1)}$ and $\boldsymbol{T}^{\boldsymbol{\prime}^{(2)}}$ based on the nonuniform density-based spatial clustering of applications with noise (DBSCAN) algorithm, and obtain the respective cluster labels $\boldsymbol{L}^{(1)}$ and $\boldsymbol{L}^{(2)}$ of perspectives 1 and 2.

Step 3. Determine the similarity between $\boldsymbol{L}^{(1)}$ and $\boldsymbol{L}^{(2)}$ based on the Jaccard coefficient. The clustering process terminates if the similarity is greater than a preestablished threshold; otherwise, continue to the next step.

Step 4. Apply LDA(linear discriminant analysis) dimensionality reduction to perspective 2 data $\boldsymbol{T}^{(2)}$ based on perspective 1 labels $\boldsymbol{L}^{(1)}$ to obtain a new subspace $\boldsymbol{T}^{\boldsymbol{\prime}^{(2)}}$, and apply LDA reduction to perspective 1 data $\boldsymbol{T}^{(1)}$ based on perspective 2 labels $\boldsymbol{L}^{(2)}$ to obtain a new subspace $T^{(1)}$. Return to step 2 .

Algorithm 1: Processing steps of the multiperspective collaborative clustering algorithm.

learning results of the data from different perspectives to modify the clustering sorting model. Accordingly, the iterative process maximizes the consistency of a given radiation source signal under different perspectives
(3) The nonuniform DBSCAN clustering method requires no initially established number of clusters in the dataset in advance, which conforms to the noncooperative characteristics of electronic reconnaissance. At the same time, the clustering effect is 


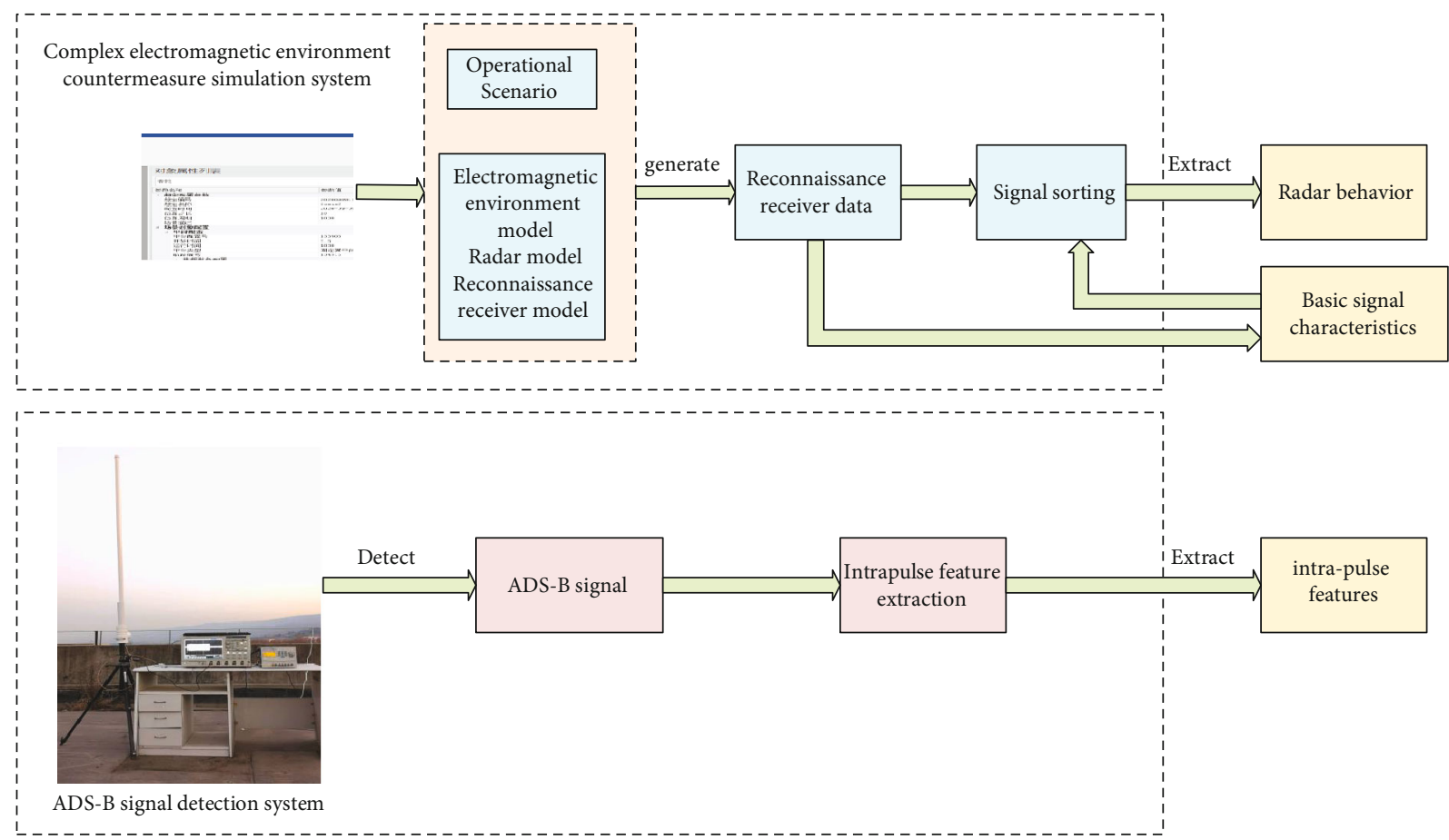

Figure 3: Experimental dataset generation method.

enhanced because it can also cluster datasets with uneven pulse densities

(4) The Jaccard coefficient is an index based on pairwise measurement and is introduced to determine the similarity between the data distributions associated with the cluster labels derived from the two perspectives. Then, the determined level of similarity is employed as the algorithm termination condition. For the distribution of the two clustering results, the Jaccard coefficient is defined as the ratio of true positive (TP) point pairs to the sum of TP and the false negative $(\mathrm{FN})$ and false positive $(\mathrm{FP})$ point pairs without considering true negative (TN) point pairs. Accordingly, the Jaccard coefficient is expressed as

$$
\text { Jaccard }=\frac{T P}{T P+F N+F P} .
$$

According to the definition, the similarity between two distributions increases as the value of the Jaccard coefficient increases up to a maximum value of 1 , and a better clustering effect is therefore obtained by the algorithm. The Jaccard coefficient threshold representing the clustering termination condition is set herein as 0.99 .

\section{Simulation Experiments}

5.1. Dataset Generation and Experimental Settings. A complex electromagnetic environment simulation system was used to generate basic signal characteristics and intrapulse feature data based on real radiation source signals, and these analog signal characteristics were extracted by a signal detec- tion system. The specific process employed for experimental data generation is presented in Figure 3.

According to the actual conditions encountered by reconnaissance receivers in the field, the number of radiation sources employed in the scene was 19, including 6 types of radiation sources: airborne fire control radar, shipborne fire control radar, surface-to-air missile target indicating radar, ground-to-air missile target indicating radar, air missile guidance radar, early warning aircraft radar, and large ground early warning radar. The different radiation sources were switched between the different signal types according to changes in the combat process. Accordingly, the number of radiation sources in the 6 categories at any given time was no less than 15 . The reconnaissance receiving equipment captured 19,331,293 pulse data from the 19 radar radiation sources over an 8 min period of system simulation. The basic characteristic data captured from the radar signals was the carrier frequency (RF), pulse width (PW), TOA, and angle of arrival (AOA), and these characteristics of the received pulse data are plotted in Figures 4-6.

To simplify the discussion presented here, the large amount of collected data was reduced by selecting only that data collected within a $2 \mathrm{~min}$ period with the most abundant number and types of pulsed radiation sources for conducting clustering and sorting. The basic signal characteristic data consisted of only the RF, PW, and pulse AOA data of 20 signal segments in the X-band with an azimuth angle of $80^{\circ}-160^{\circ}$, and the number of pulses in each signal segment was 30,000. In addition, the intrapulse feature data included the instantaneous feature and bispectral feature data extracted from the leading edge of an automatic dependent surveillancebroadcast (ADS-B) signal pulse of a civil aviation aircraft. 


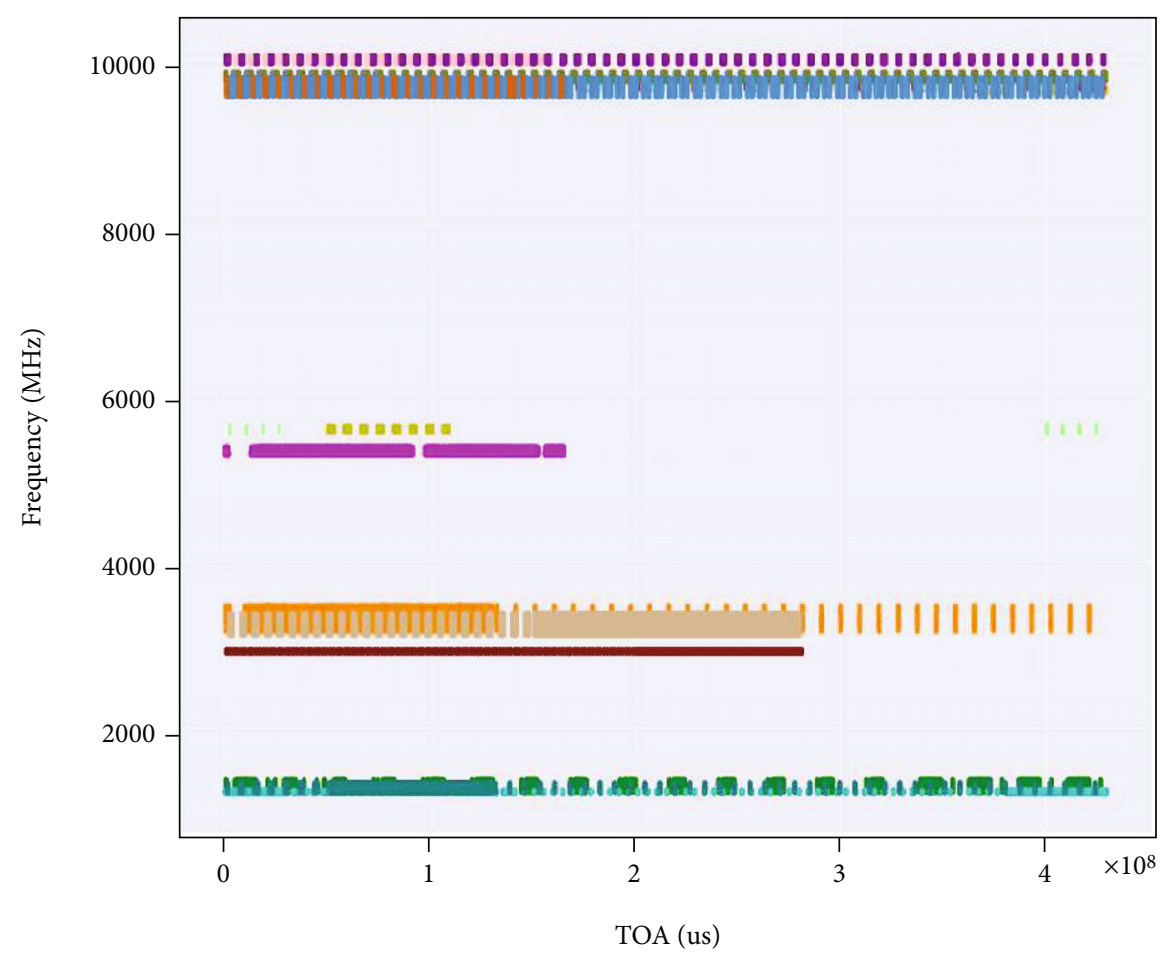

FIgURE 4: Carrier frequency (RF) versus the pulse time of arrival (TOA) for the received data.

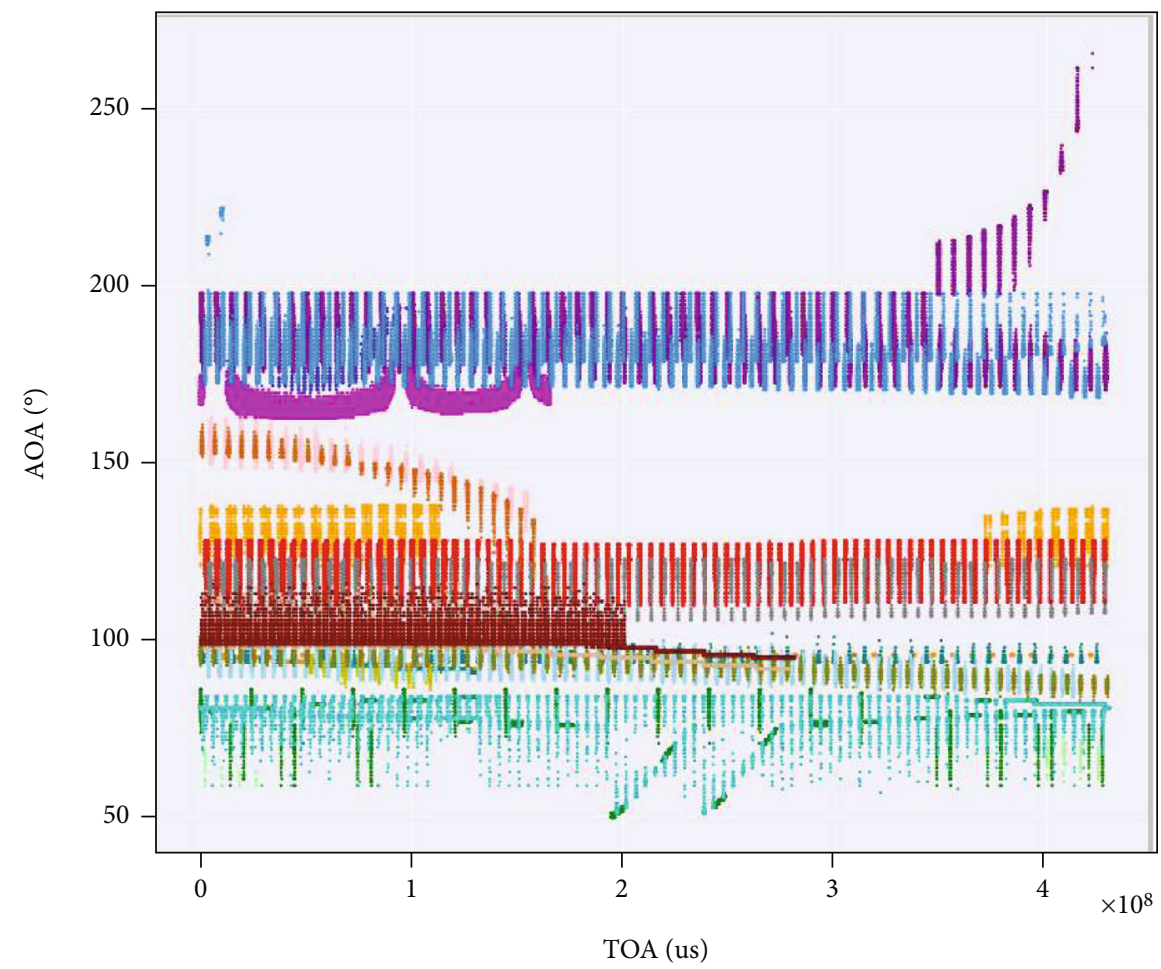

FIgURE 5: Pulse angle of arrival (AOA) versus pulse TOA for the received data.

The extraction time of the pulse front was $0.2 \mu \mathrm{s}$, and the instantaneous and bispectral feature data are, respectively, presented in Figures 7 and 8. After feature splicing, the dimensionality of the intrapulse features at the leading edge of each pulse signal was 140 dimensions. The instantaneous features of the signal and the number of segmented cuts of the integral bispectrum were 3 segments, and the variance, kurtosis, skewness, permutation entropy, and fuzzy entropy were extracted for each feature sequence and for the entire feature sequence. The 


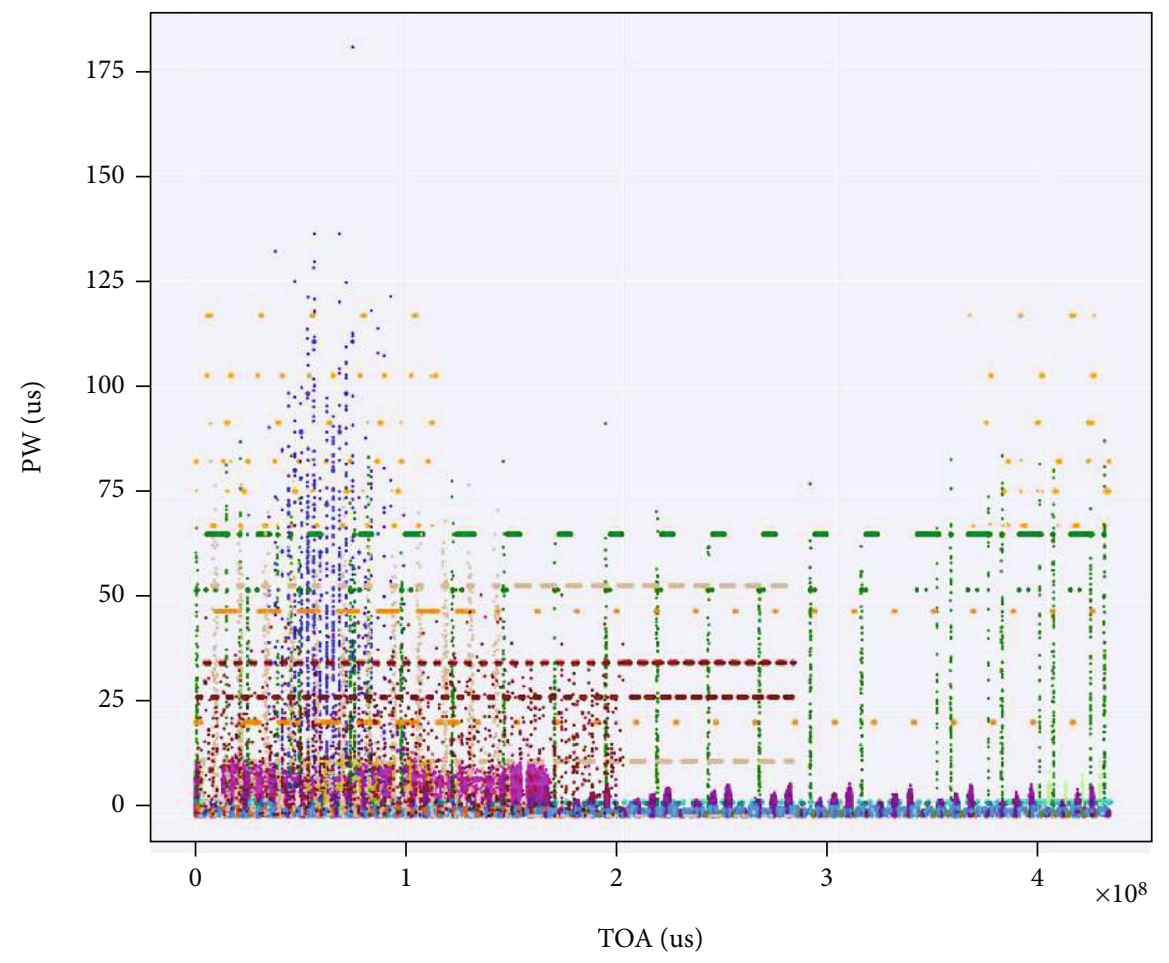

FIgure 6: Pulse width (PW) versus TOA for the received data.

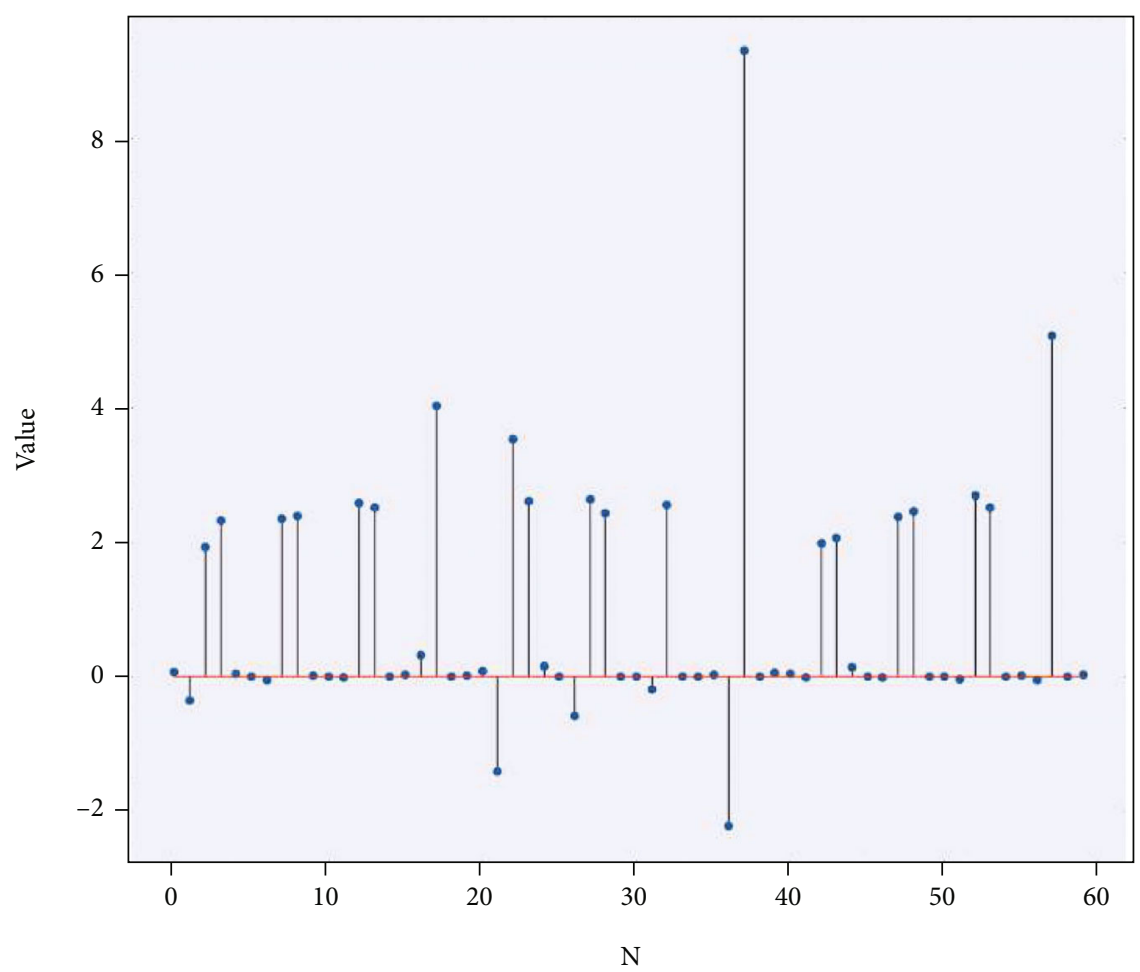

Figure 7: Schematic illustrating the instantaneous features of intrapulse signals.

intrapulse feature data was reduced to 10 dimensions using KPCA dimensionality reduction. Accordingly, the sklearn function setting was set to kernel = 'rbf' and $\mathrm{n}_{-}$ components $=10$. Because the basic signal characteristic data included only 3 dimensions, this data was not reduced in dimensionality. Therefore, the PCA parameter setting included $\mathrm{n}_{-}$components $=3$.

5.2. Dimensionality Reduction Analysis of Intrapulse Features. Figure 9 presents the KPCA dimensionality 


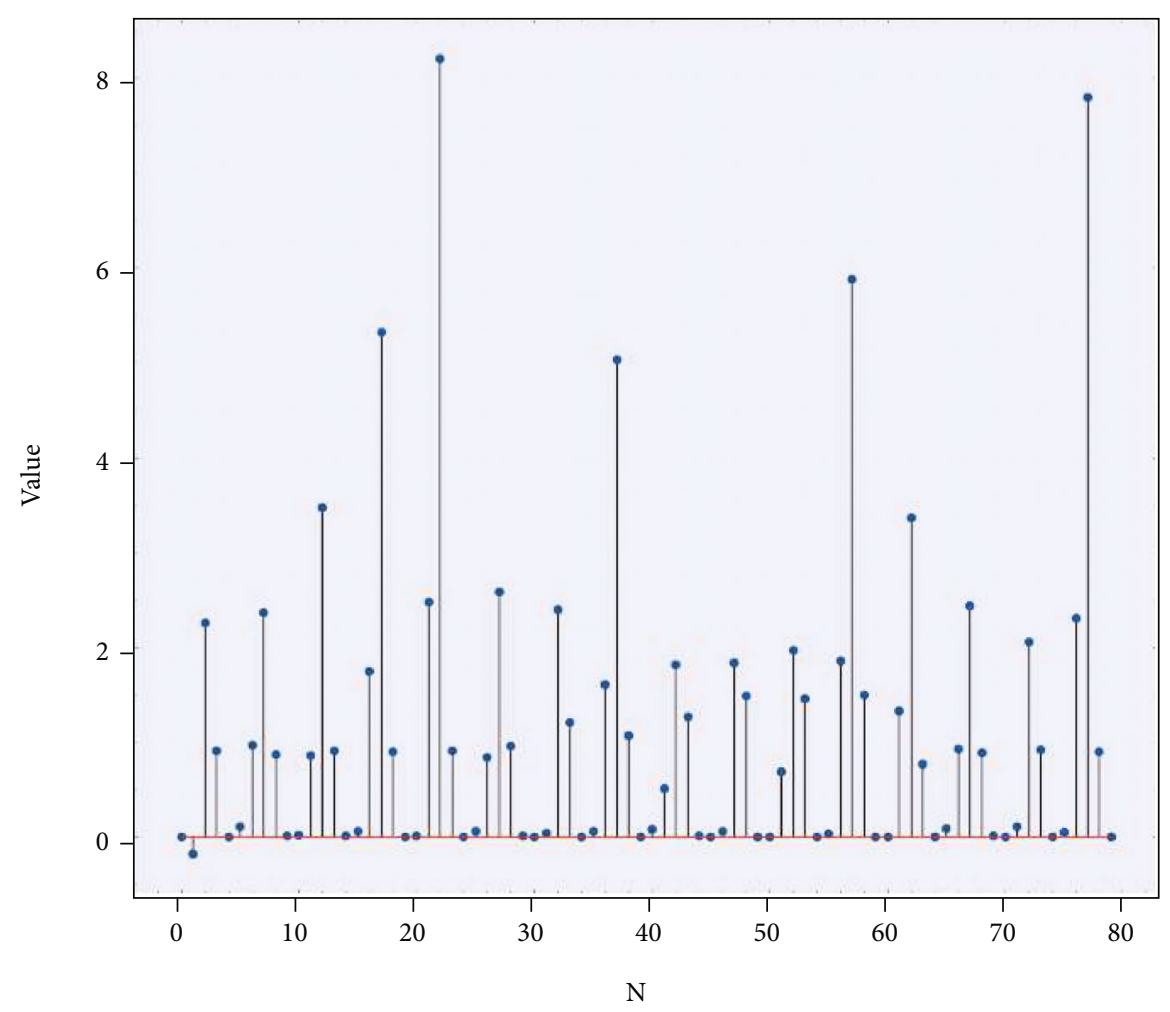

FIGURE 8: Schematic illustrating the bispectral features of intrapulse signals.

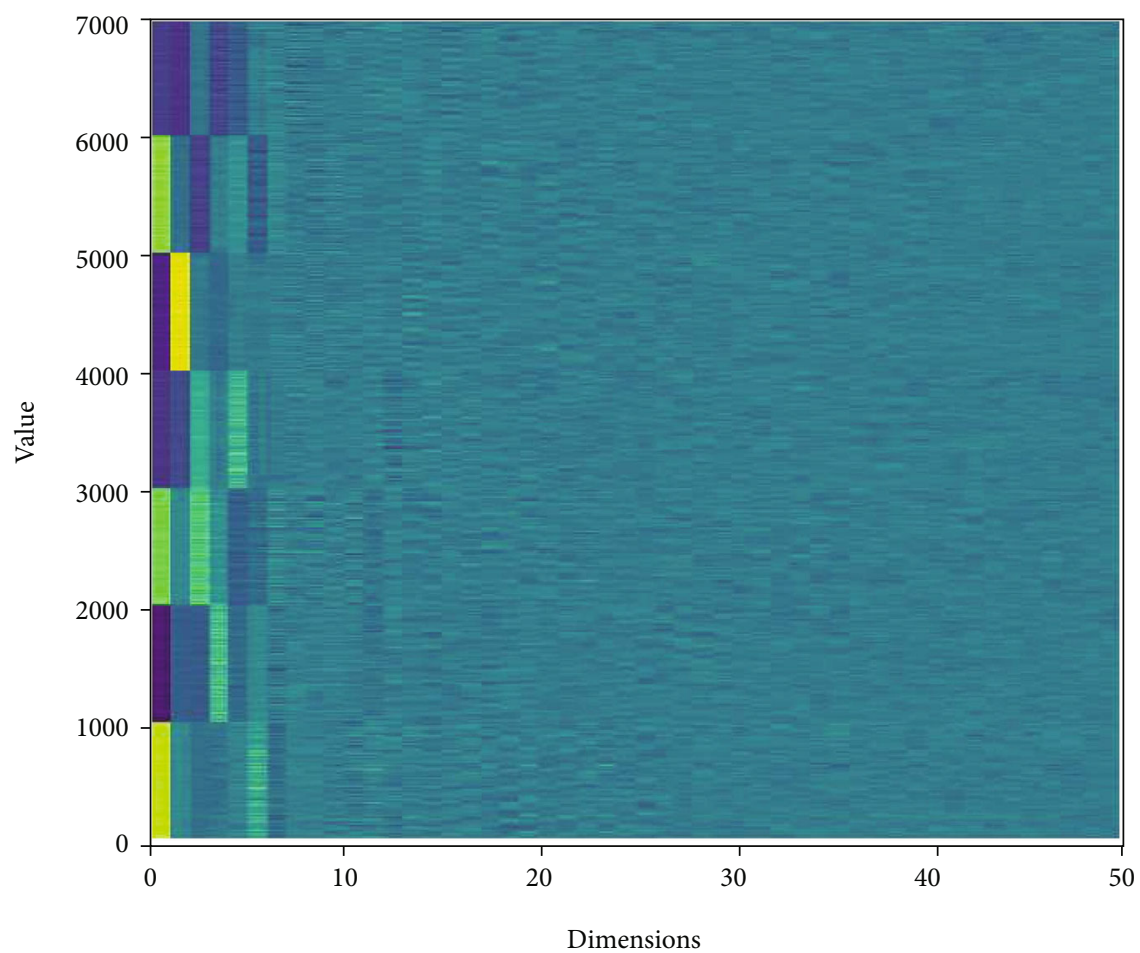

FIgURE 9: Dimensionality reduction distribution of the pulse features of different radiation sources (reduced dimension $=50)$.

reduction distribution of 7 radiation source signals to 50 dimensions, where the number of samples for each radiation source was 1000. It can be seen that the features of the different radiation sources within the pulse are reduced after dimensionality reduction, and the main differences between the different radiation sources are concentrated in the most principal components. The eigenvalues of each principal component are analyzed in Figure 10. It can be seen that 


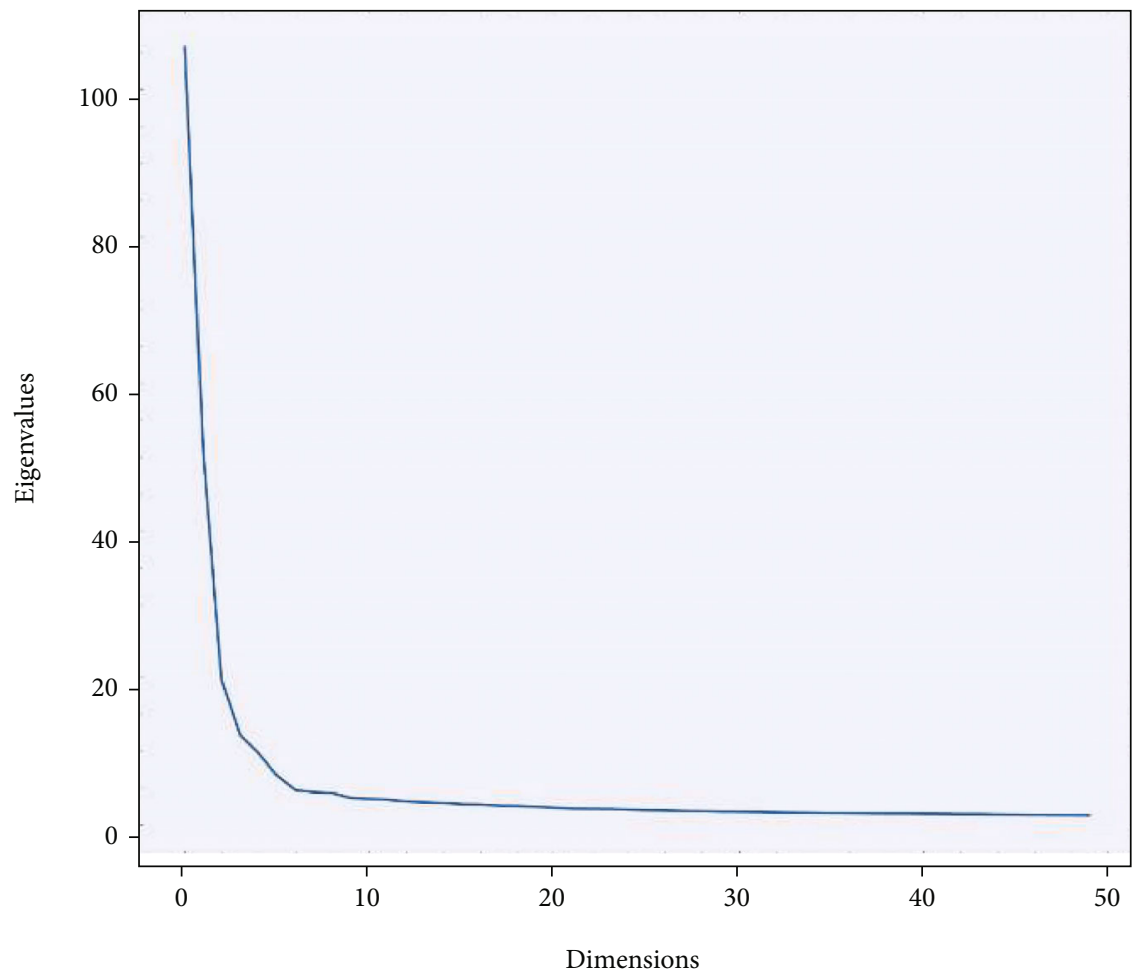

Figure 10: Distribution of eigenvalues after dimensionality reduction for the pulse features of different radiation sources (reduced dimension $=50$ ).

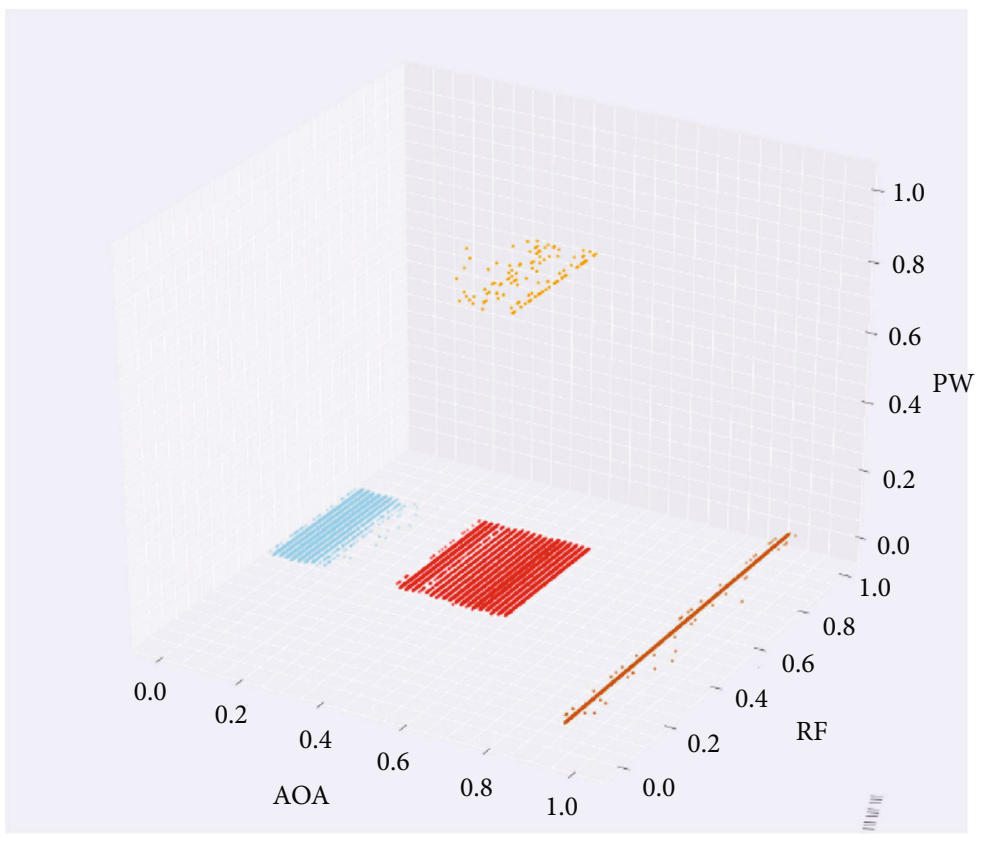

Figure 11: Original basic signal characteristic data distributions of segment 10.

the feature value decreases sharply when the number of principal components exceeded 5 , and the cumulative contribution to the variance of the first 5 principal components was $66.4 \%$. Therefore, the intrapulse features were reduced to 5 dimensions by the KPCA method in subsequent experiments. The dimensionality reduction ensures that the feature distributions of the different radiation sources are different, which enables the signals of different radiation sources to be effectively distinguished for realizing signal sorting.

5.3. Comparison of Clustering and Sorting Algorithms. The clustering performance of the multiperspective collaborative clustering sorting method was compared with those of the 


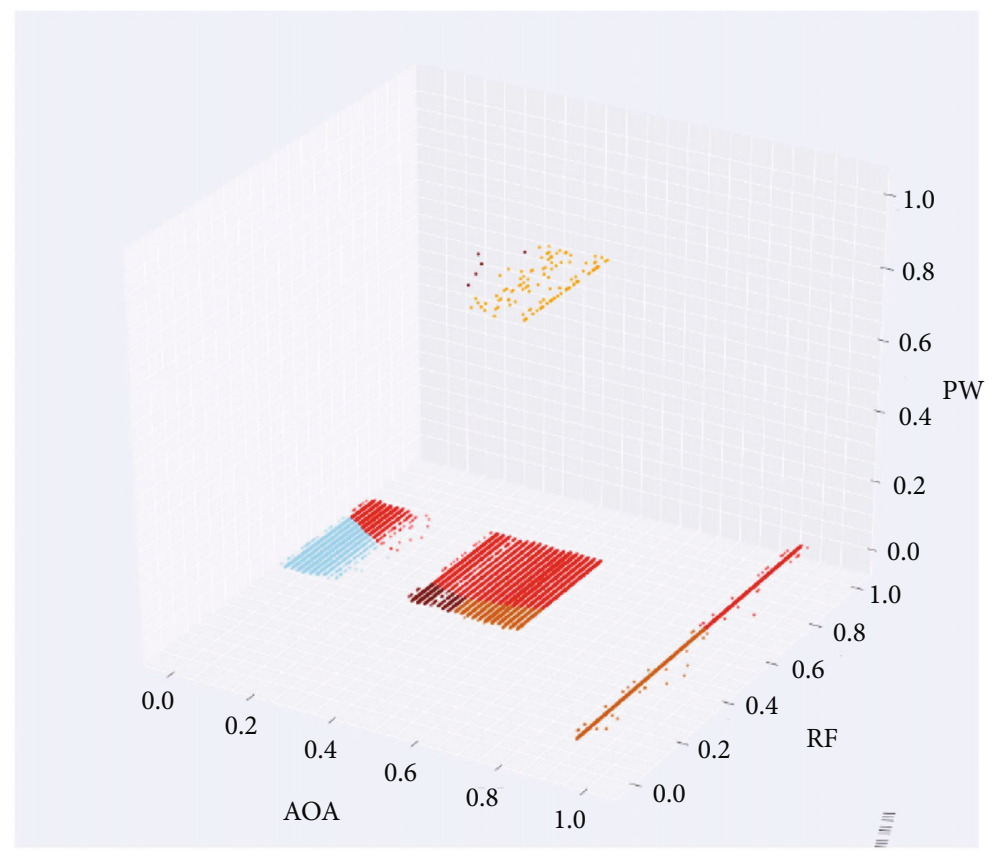

FIgURE 12: Data field grid clustering and sorting results.

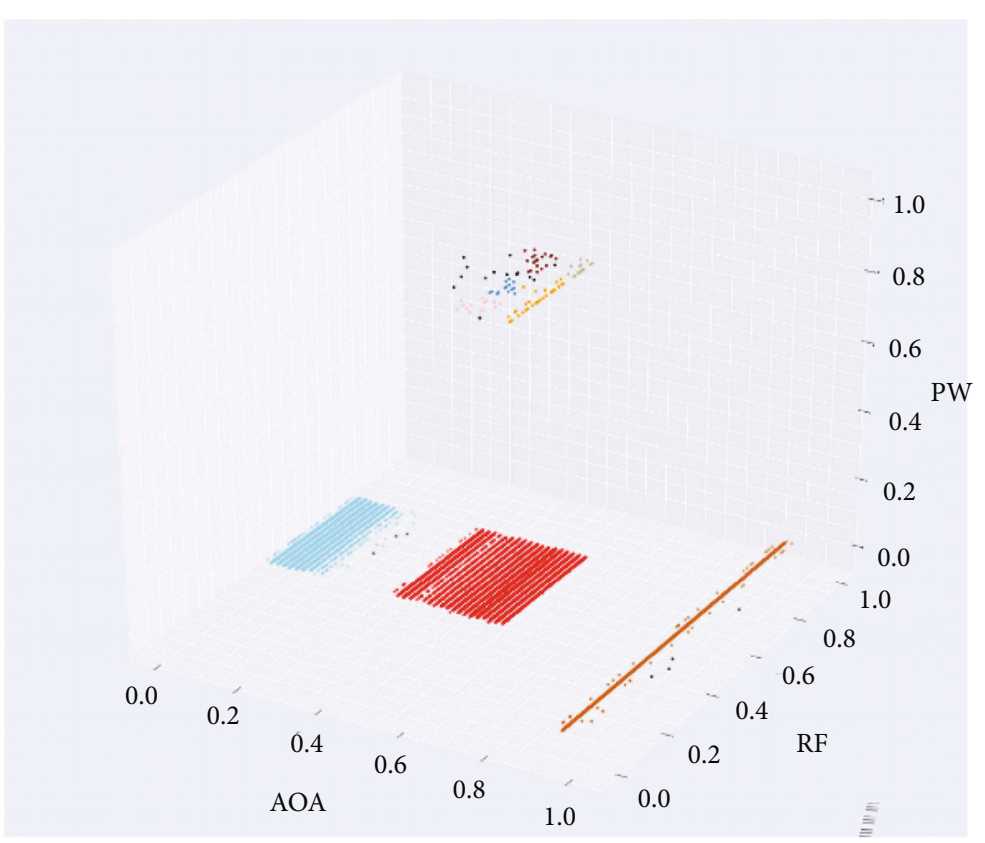

FIGURE 13: DBSCA clustering and sorting results.

data field grid clustering algorithm and the DBSCAN algorithm. Figure 11 presents segment 10 of the original data, which included only 4 radiation sources, and Figures 1214 , respectively, present the corresponding clustering results of the data field grid (Figure 12), DBSCAN (Figure 13), and the proposed clustering and sorting algorithms (Figure 14). The data application of DBSCAN algorithm and data field grid clustering algorithm is the basic characteristics of the signal. Here, the basic signal characteristic data (i.e., AOA, $\mathrm{RF}$, and PW) have been normalized to simplify the plots along values ranging from 0 to 1.0.
From Figures $12-14$, it can be seen that the data field grid and DBSCAN clustering and sorting algorithms present obvious clustering errors. This is particularly the case for the data field grid algorithm, which presents a relatively large number of clusters owing to the box-like distribution of the collected data that can support multiple potential centers. Here, the 30,000 data points from the 4 radiation sources are classified into 6 categories. The use of a globally fixed neighborhood radius by the DBSCAN clustering algorithm introduces clustering errors for those data points with uneven density at high PW. In contrast, we note that the 


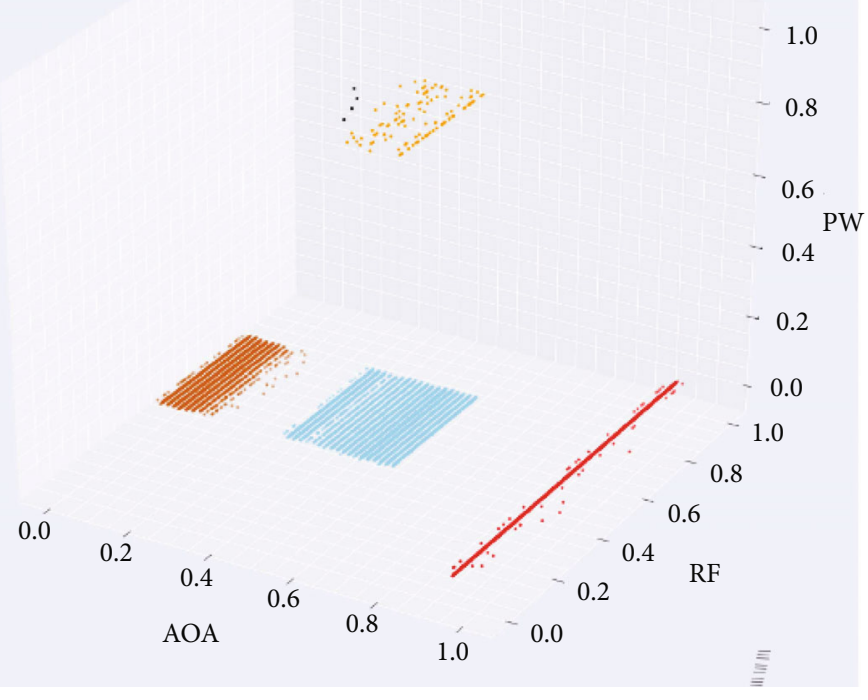

FIGURE 14: Results of the multiperspective collaborative clustering and sorting method.

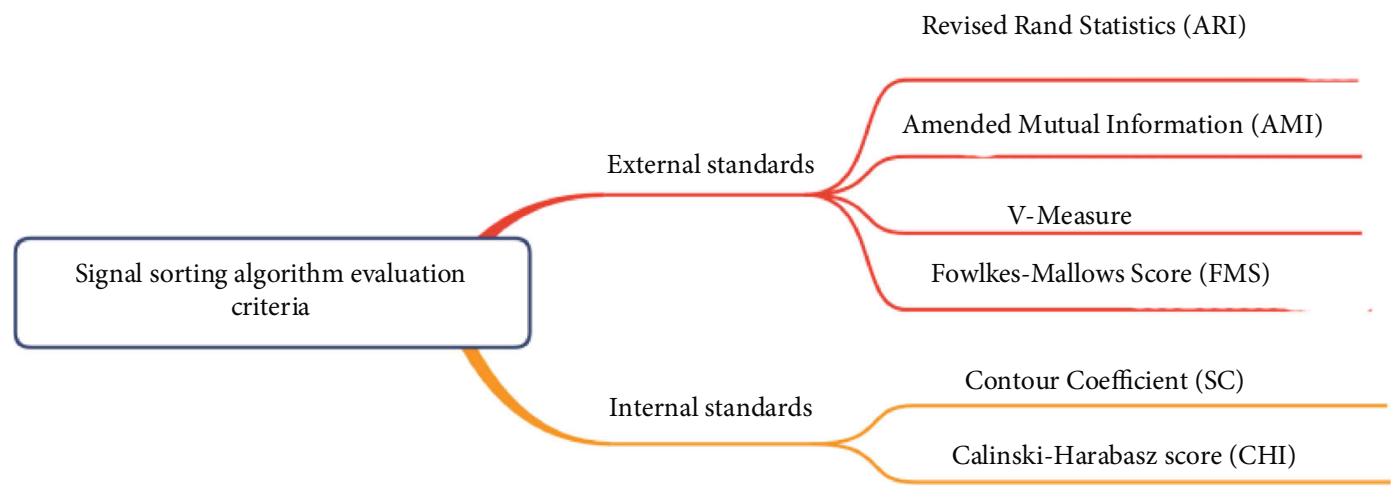

Figure 15: Evaluation index system applied to clustering and sorting algorithms.

TABLE 1: Comparison of the performances of different clustering and sorting algorithms.

\begin{tabular}{lccccrr}
\hline Algorithm & ARI & AMI & V-measure & FMS & SC & CHI \\
\hline Data field grid clustering & 0.7751 & 0.8178 & 0.8179 & 0.8946 & 0.5830 & 90332 \\
DBSCAN & 0.9218 & 0.8994 & 0.8994 & 0.9723 & 0.6041 & 60088 \\
Multiperspective collaborative clustering & 0.9873 & 0.9783 & 0.9783 & 0.9926 & 0.7199 & 90682 \\
\hline
\end{tabular}

TABLE 2: Comparison of multiperspective and single-perspective clustering and sorting experiments.

\begin{tabular}{lccccrr}
\hline Algorithm & ARI & AMI & V-measure & FM-score & SC & CHI \\
\hline Single perspective nonuniform DBSCAN & 0.9503 & 0.9221 & 0.9221 & 0.9746 & 0.5692 & 68381 \\
Multiple perspective nonuniform DBSCAN & 0.9873 & 0.9783 & 0.9783 & 0.9926 & 0.7199 & 90682 \\
\hline
\end{tabular}

clustering effect of the proposed multiperspective collaborative clustering and sorting algorithm is best. Here, the basic characteristic data are correctly clustered, except for four discrete data points at high PW.
The performances of the clustering and sorting algorithms were further evaluated comprehensively by means of indices composed of 4 external standards and 2 internal standards. For all evaluation standards considered, the 


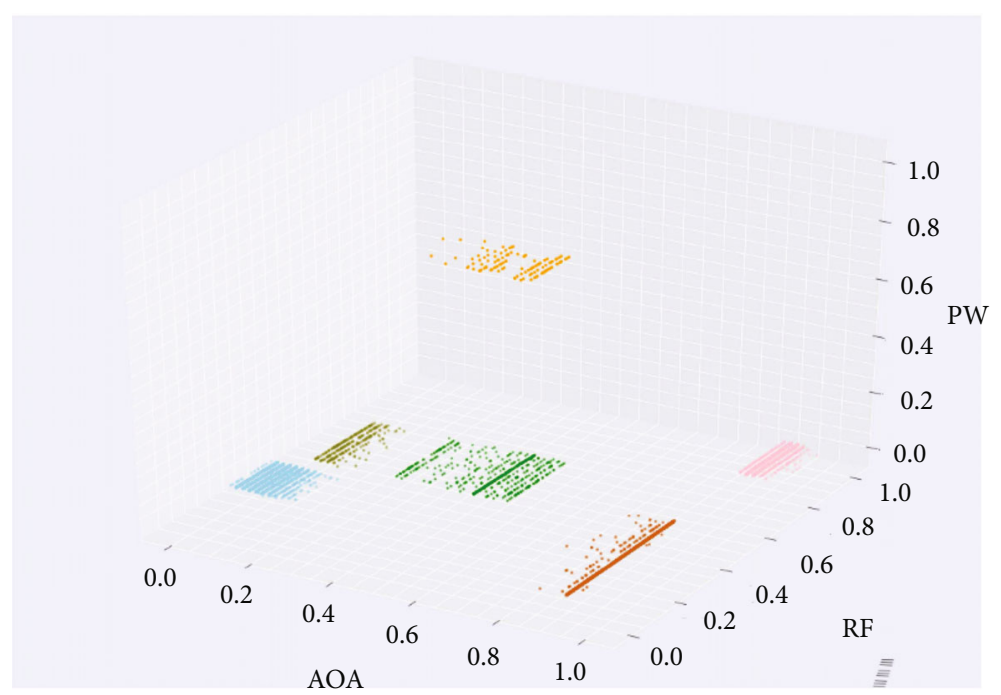

FIgURE 16: True distribution of segment 6 data based on perspective 1 .

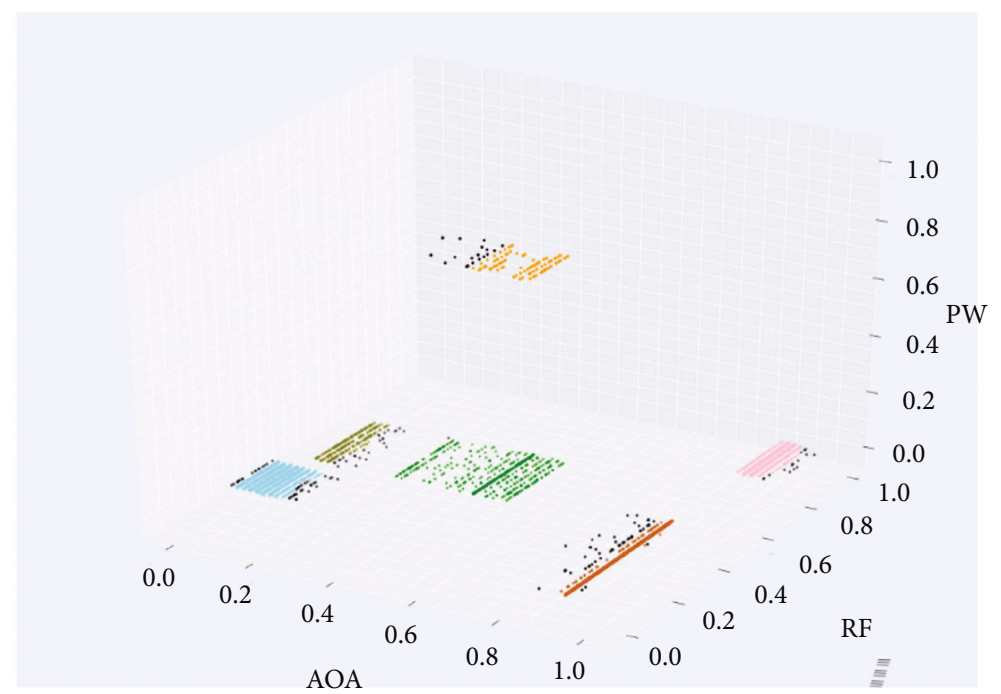

Figure 17: Initial clustering distribution of segment 6 data based on perspective 1 .

performance of an algorithm increases with increasing index value. The evaluation index system employed is illustrated in Figure 15, and the results of analysis are listed in Table 1. It can be seen from the table that the proposed multiperspective collaborative clustering and sorting algorithm provides superior performance for all evaluation standards considered.

The effectiveness of the multiperspective collaborative clustering approach proposed in this paper was further verified by comparing the performance of the nonuniform DBSCAN clustering algorithm employed in the proposed approach under both single-perspective and multipleperspective frameworks. The performance results are listed in Table 2. As can be seen from the table, the clustering results obtained by the nonuniform DBSCAN clustering algorithm under the multiperspective framework are superior to that obtained under the single-perspective framework.
5.4. Clustering and Sorting Process Analysis of the Proposed Algorithm. The multiperspective collaborative clustering and sorting process was further analyzed by evaluating the changes in the clustering results of the radiation source data of segment 6 from its original distribution, which included 6 radiation sources, to the initial clustering results and those obtained over three successive iterations, whereas discussed above, data is learned from the two different perspectives alternately, and the difference between the learning results of the data from the two perspectives is employed to modify the clustering sorting model. The various distributions of the segment 6 data are presented in Figures 16-20 based on perspective 1 . Here, the actual distribution of segment 6 data is presented in Figure 16, while Figure 17 presents the initial clustering results, and Figures 18-20 illustrate the evolution in the clustering results after three collaborative clustering iterations. 


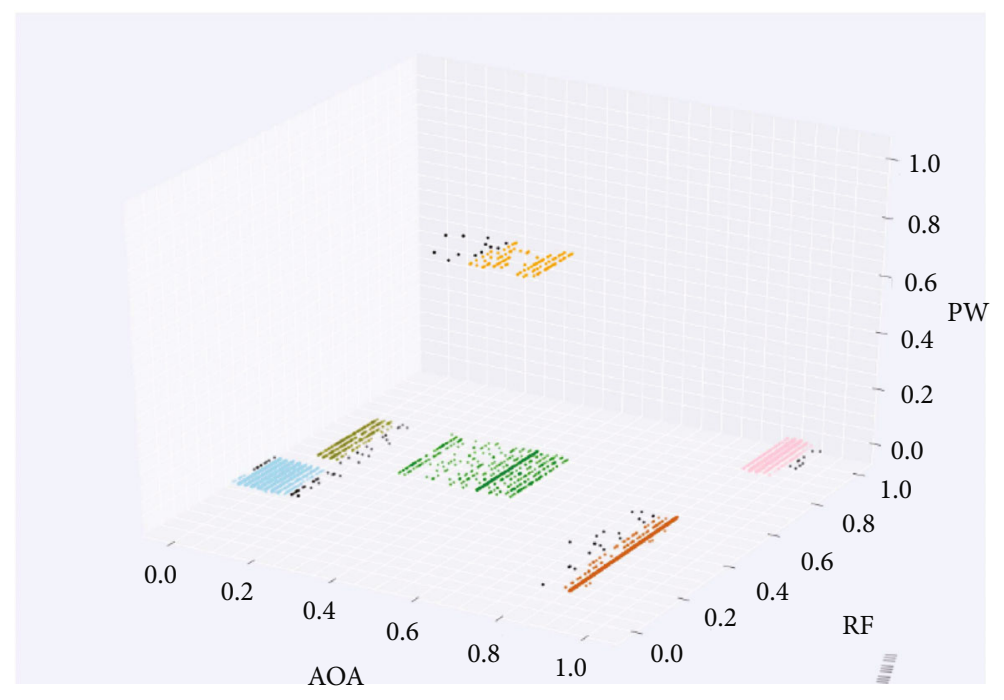

FIGURE 18: First collaborative clustering results of segment 6 data based on perspective 1 .

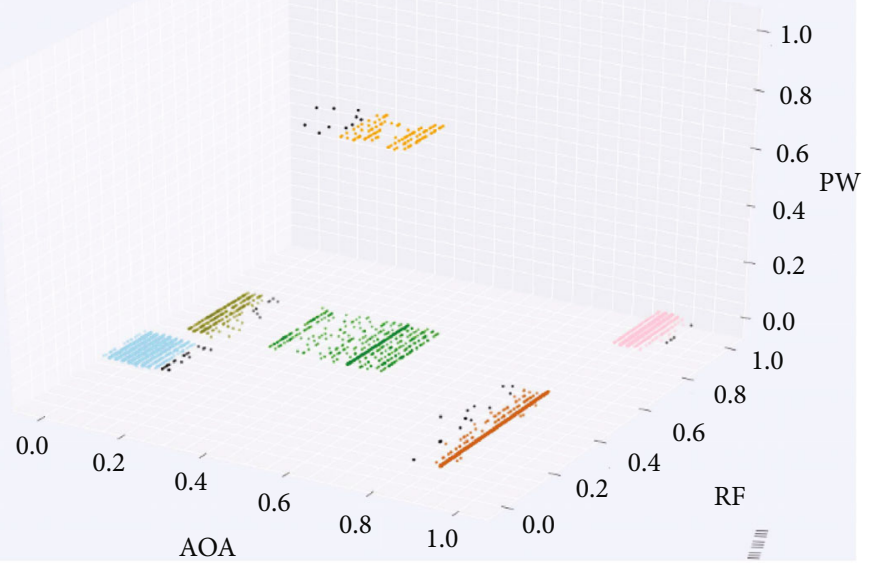

FIGURE 19: Second collaborative clustering results of segment 6 data based on perspective 1 .

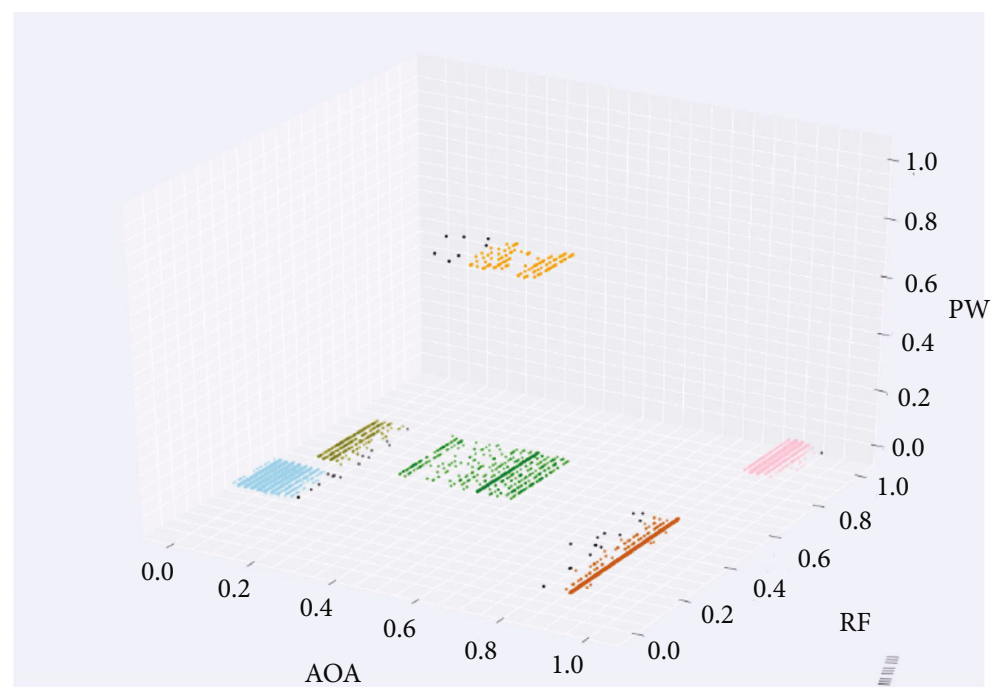

FIgURE 20: Third collaborative clustering results of segment 6 data based on perspective 1 . 
The collaborative optimization process shown in Figures $17-20$ demonstrates that the clustering accuracy is gradually improved with successive iterations. This is because the data derived from the two different perspectives describe the target attributes differently. As a result, the cluster label transfer and LDA dimensionality reduction processes, respectively, correct the clustering model and generate new subspaces, under which, the clustering results of the unsupervised clustering algorithm are improved.

\section{Conclusion}

The present study addressed the disadvantages of currently available cognitive electronic reconnaissance methods under the complex electromagnetic environments on modern battle fields by proposing a multiperspective collaborative clustering method for sorting radiation sources based on the multiperspective information of radar signals. The two perspectives employed include the basic signal characteristics and intrapulse features of radar signals. The proposed method performs unsupervised clustering, cluster label transfer, and PCA dimensionality reduction iteratively based on the differences between the clustering results obtained from the two signal perspectives. Therefore, radiation signal sorting can be conducted in a noncooperative context without human supervision. The common problems associated with the high dimensionality and correlation characteristics of the intrapulse features of radar signals are addressed by applying nonlinear dimensionality reduction using KPCA. The results of comparative experiments demonstrate that the proposed multiperspective sorting method can make full use of the difference information between the two signal perspectives and thereby improve the accuracy of clustering-based radiation source sorting. Application of the multiperspective clustering method to actual radar signals in a realistically complex electromagnetic environment demonstrated its capability of improving the radar signal sorting results of cognitive electronic reconnaissance systems compared with other state-of-the-art clustering methods. Moreover, the multiperspective collaborative clustering sorting method is demonstrated to provide a better clustering and sorting ability than the single-perspective clustering method. However, in the simulation process, it is found that the data processing time is long, and reducing the complexity of the algorithm in the later stage is one of the problems that needs to be solved.

\section{Data Availability}

The data that support the findings of this study are available upon request from the corresponding author Y.Z. The data are not publicly available due to restrictions, e.g., their containing information that could compromise the privacy of research participants.

\section{Conflicts of Interest}

The authors declare no conflict of interest.

\section{Acknowledgments}

The author would like to thank the Shaanxi Provincial Science and Nature Foundation (approval number 2021JM225) for providing funds for the experiment.

\section{References}

[1] S. Q. Wang, G. P. Hu, Q. L. Zhang, C. Y. Gao, and T. Cai, "The background and significance of radar signal sorting research in modern warfare," Procedia Compute Science, vol. 154, pp. 519$523,2019$.

[2] H. K. Mardia, "New techniques for the deinterleaving of repetitive sequences," IEE Proceedings F (Radar and Signal Processing), vol. 136, no. 4, pp. 149-154, 1989.

[3] D. Nelson, "Special purpose correlation functions for improved signal detection and parameter estimation," in Proceedings Icassp IEEE International Conference on Acoustics Speech \& Signal Processing, vol. 4, pp. 73-76, Minneapolis, MN, USA, 1993.

[4] V. Chandra and R. C. Bajpai, "ESM data processing parametric deinterleaving approach," in TENCON'92 - Technology Enabling Tomorrow, pp. 26-30, Melbourne, VIC, Australia, 1992.

[5] C. Cheng and J.-Y. Tourneret, "An EM-based multipath interference mitigation in GNSS receivers," Signal Processing, vol. 162, pp. 141-152, 2019.

[6] X. Gong, K. Cao, P. Jia, and G. Shangfu, "K-modes algorithm based on rough set and information entropy," Journal of Physics: Conference Series, vol. 1754, no. 1, p. 1754, 2021.

[7] X. Shou-Kun, W. Chao, Z. Li-Hua, and G. Xin-Hua, "DBSCAN clustering algorithm for the detection of nearby open clusters based on Gaia-DR2two," Chinese Astronomy and Astrophysics, vol. 43, no. 2, pp. 225-236, 2019.

[8] F. Digne, A. Baussare, C. Cornu, and D. Jahan, "Classification of radar pulses in a naval warfare context using Bezier curve modeling of the instantaneous frequency law," IEEE Transactions on Aerospace and Electronic Systems, vol. 53, no. 3, article 2671578, 2017.

[9] Y. Zhao, L. Wu, J. Zhang, and Y. Li, "Specific emitter identification using geometric features of frequency drift curve," Bulletin of the Polish Academy of Science Technical Sciences, vol. 66, no. 1, pp. 99-108, 2018.

[10] X. Ru, Z. Huang, Z. Liu, and W. Jiang, "Frequency-domain distribution and band-width of unintentional modulation on pulse," Electronics Letters, vol. 52, no. 22, pp. 1853-1855, 2016.

[11] J. Han, T. Zhang, and Z. Qiu, "Communication emitter individual identification via $3 \mathrm{D}$-Hilbert energy spectrum-based multiscale segmentation features," International Journal of Communication Systems, vol. 32, no. 1, article e3833, 2019.

[12] R. W. Klein, M. A. Temple, and M. J. Mendenhall, "Application of wavelet-based RF fingerprinting to enhance wireless network security," Journal of Communications and Networks, vol. 11, no. 6, pp. 544-555, 2009.

[13] T. J. Bihl, K. W. Bauer, and M. A. Temple, "Feature selection for RF fingerprinting with multiple discriminant analysis and using ZigBee device emissions," IEEE Transactions on Information Forensics and Security, vol. 11, no. 8, pp. 1862-1874, 2016.

[14] J. Dudczyk and A. Kawalec, "Identification of emitter sources in the aspect of their fractal features," Bulletin of the Polish 
Academy of ences Technical ences, vol. 61, no. 3, pp. 623-628, 2013.

[15] J. Dudczyk and A. Kawalec, "Specific emitter identification based on graphical representation of the distribution of radar signal parameters," Bulletin of the Polish Academy of Sciences. Technical Sciences, vol. 63, no. 2, pp. 391-396, 2015.

[16] J. Dudczyk, "A method of feature selection in the aspect of specific identification of radar signals," Bulletin of the Polish Academy of ences Technical ences, vol. 65, no. 1, pp. 113-119, 2017.

[17] S. Haykin, "Cognitive radar: a way of the future," IEEE Signal Processing Magazine, vol. 23, no. 1, pp. 30-40, 2006.

[18] S. Haykin, Cognitive radar Knowledge-based radar detection, tracking, and classification, John Wiley \& Sons, Ltd, 2007.

[19] V. K. Chaurasia and H. Amhia, "Reduction of impulse noise by using K-mean clustering segmentation approach," in 2019 International Conference on Communication and Signal Processing (ICCSP), pp. 0690-0694, Chennai, India, 2019.

[20] P. K. Guru Diderot, N. Vasudevan, and K. S. Sankaran, “An efficient fuzzy C-means clustering based image dissection algorithm for satellite images," in 2019 International Conference on Communication and Signal Processing (ICCSP), pp. 08060809, Chennai, India, 2019.

[21] M. Ren, J. Duan, and S. Yang, "An ambiguity function transformation characteristic for radar signal sorting," Journal of Physics: Conference Series, vol. 1738, no. 1, pp. 012034012044, 2021.

[22] "A new clustering and sorting algorithm for radar emitter signals," Journal of Physics: Conference Series, vol. 1617, no. 1, pp. 012009-012020, 2020.

[23] R. Cao, J. Cao, J. P. Mei, C. Yin, and X. Huang, "Radar emitter identification with bispectrum and hierarchical extreme learning machine," Multimedia Tools \& Applications, vol. 78, no. 20, pp. 28953-28970, 2018.

[24] S. Wang, C. Gao, Y. Zhang, Q. Zhang, H. Zeng, and J. Bai, "Radar signal sorting method based on support vector clustering and grey correlation degree index," International Journal of Information and Communication Technology, vol. 16, no. 4, pp. 353-364, 2020.

[25] J. Matuszewski and D. Pietrow, "Recognition of electromagnetic sources with the use of deep neural networks," in XII Conference on Reconnaissance and Electronic Warfare Systems, Oltarzew, Poland, 2019.

[26] Z. Zhou, G. Huang, H. Chen, and J. Gao, “Automatic radar waveform recognition based on deep convolutional denoising auto-encoders," Circuits, Systems, and Signal Processing, vol. 37, no. 9, pp. 4034-4048, 2018.

[27] A. V. Kvasnow, "Methodology of classification and recognition of the radar emission sources based on Bayesian programming," IET Radar, Sonar and Navigation, vol. 14, no. 8, pp. 1175-1182, 2020.

[28] S. Bhattacharya, P. K. R. Maddikunta, I. Meenakshisundaram et al., "Deep neural networks based approach for battery life prediction," CMC-COMPUTERS MATERIALS \& CONTINUA, vol. 69, no. 2, pp. 2599-2615, 2021.

[29] W. Bin, Y. Shibo, and L. Peng, "Radar emitter signal recognition based on one-dimensional convolutional neural network with attention mechanism," Sensors, vol. 20, no. 21, pp. 6350-6350, 2020.

[30] C. Iwendi, P. K. R. Maddikunta, T. R. Gadekallu, K. Lakshmanna, A. K. Bashir, and M. J. Piran, "A Metaheuristic Optimization Approach for Energy Efficiency in the IoT
Networks," Software: Practice and Experience, vol. 51, no. 12, pp. 2558-2571, 2020.

[31] A. Blum and T. Mitchell, "Combining labeled and unlabeled data with co-training," in Proceedings of the 11th annual Conference on Computational Learning Theory, Madison, Wisconsin, USA, 1998.

[32] K. Nigam and R. Ghani, "Analyzing the effectiveness and applicability of co-training," in Proceedings of the ninth international conference on Information and knowledge management - CIKM '00, Virginia, McLean, USA, 2000.

[33] M. F. Balcan, A. Blum, and K. Yang, "Co-training and expansion: towards bridging theory and practice," in Advances in Neural Information Processing Systems, Vancouver, British Columbia, Canada, December 2004.

[34] C. Feng, K. Yu, M. Aloqaily, M. Alazab, Z. Lv, and S. Mumtaz, "Attribute-based encryption with parallel outsourced decryption for edge intelligent IoV," IEEE Transactions on Vehicular Technology, vol. 69, no. 11, p. 13784-13795, 2020.

[35] X. L. Shao, Y. Shi, and W. D. Zhang, "Input-and-measurement event-triggered output-feedback chattering reduction control for MEMS gyroscopes," IEEE Transactions on Systems, Man, and Cybernetics: Systems, vol. 12, pp. 1-12, 2021.

[36] M. Chen, K. Q. Weinberger, and J. Blitzer, "Co-training for domain adaptation," in Advances in neural information processing systems 24, Curran Associates Inc., 2011.

[37] X. L. Shao, Y. Shi, and W. D. Zhang, "Fault-tolerant quantized control for flexible air-breathing hypersonic vehicles with appointed-time tracking performances," IEEE Transactions on Aerospace and Electronic Systems, vol. 57, no. 2, pp. 12611273, 2021.

[38] X. H. Yue, X. L. Shao, and W. D. Zhang, "Elliptical encircling of quadrotors for a dynamic target subject to aperiodic signals updating," Ieee Transactions on Aerospace and Electronic Systems, 2021. 